\title{
The real population of star clusters in the bar of the Large Magellanic Cloud ${ }^{\star}$
}

\author{
Andrés E. Piatti ${ }^{1,2}$ \\ 1 Consejo Nacional de Investigaciones Científicas y Técnicas, Av. Rivadavia 1917, C1033AAJ Buenos Aires, Argentina \\ 2 Observatorio Astronómico, Universidad Nacional de Córdoba, Laprida 854, 5000 Córdoba, Argentina \\ e-mail: andres@oac.unc.edu.ar
}

Received 25 May 2017 / Accepted 13 July 2017

\begin{abstract}
We report results on star clusters located in the south-eastern half of the Large Magellanic (LMC) bar from Washington $C T_{1}$ photometry. Using appropriate kernel density estimators, we detected 73 star cluster candidates, three of which do not show any detectable trace of star cluster sequences in their colour-magnitude diagrams (CMDs). We did not detect the other 38 previously catalogued clusters, which could not be recognized when visually inspecting the $C$ and $T_{1}$ images either; the distribution of stars in their respective fields do not resemble that of a stellar aggregate. They represent $\sim 33$ per cent of all catalogued objects located within the analysed LMC bar field. From matching theoretical isochrones to the cluster CMDs cleaned from field star contamination, we derived ages in the range $7.2<\log \left(t \mathrm{yr}^{-1}\right)<10.1$. As far as we are aware, this is the first time that homogeneous age estimates based on resolved stellar photometry are obtained for most of the studied clusters. We built the cluster frequency (CF) for the surveyed area, and found that the main star cluster formation activity has taken place during the period $\log \left(t \mathrm{yr}^{-1}\right) \sim 8.0-9.0$. Since $\sim 100$ Myr ago, clusters have been formed during a few bursting formation episodes. When comparing the observed CF to that recovered from the star formation rate, we found noticeable differences, which suggests that field star and star cluster formation histories could have been significantly different.
\end{abstract}

Key words. techniques: photometric - galaxies: individual: LMC - Magellanic Clouds

\section{Introduction}

Although it is expected that most of the Large Magellanic Cloud (LMC) clusters catalogued by Bica et al. (2008, hereafter B08,) are real extended objects, B08 did not confirm their nature. Because they come from inspection of photographic plates by eye or by automatic codes, we should not rule out that some of them could be asterisms. Piatti \& Bica (2012) and Piatti (2014b) found $10-15 \%$ of the catalogued objects to be possibly non-physical systems. Cleaning cluster catalogues is not an exciting task. Nayak et al. (2016) have preferred not to study star clusters on the basis of variation in the field star distribution or embedded in fields suffering from large dispersion in the field star count with respect to the average, around the cluster. Here, we study star clusters located in the south-eastern half of the LMC bar near the old globular cluster NGC 1939. Of the regions populated by star clusters in the galaxy, this is one of the most densely populated areas, and most of the clusters have not been studied from resolved stellar photometry so far.

The paper is organized as follows: Sect. 2 describes the data set and the procedures we used to obtain standardized Washington $C T_{1}$ photometry. We describe the search for star clusters performed from the photometric data set in Sect. 3, while in Sect. 4 we derive cluster ages. The analysis of the derived ages is carried out in Sect. 5, where we introduce the intrinsic cluster formation

\footnotetext{
* Photometric catalogues of the studied star clusters are only available at the CDS via anonymous ftp to cdsarc.u-strasbg.fr (130.79.128.5) or via http://cdsarc.u-strasbg.fr/viz-bin/qcat?]/A+A/606/A21
}

history for the surveyed region. Finally, Sect. 6 summarizes the main outcomes of this work.

\section{Observational data}

We took advantage of $C T_{1}$ Washington images available at the National Optical Astronomy Observatory (NOAO) Science Data Management (SDM) Archives ${ }^{1}$, which were obtained as part of a survey of the most metal-poor stars outside the Milky Way (CTIO 2008B-0296 programme, PI: Cole). The images analysed here consist of a $420 \mathrm{~s} C$ and a $30 \mathrm{~s} R$ exposures obtained with the $8 \mathrm{~K} \times 8 \mathrm{~K} \mathrm{CCD}$ camera $\left(36^{\prime} \times 36^{\prime}\right.$ field $)$ attached at the $4 \mathrm{~m}$ Blanco telescope (CTIO) under photometric conditions (seeing values are between 1.0 and 1.3, with an average of 1.1) and at an airmass of 1.3 .

The data sets were fully processed following the procedures extensively described in Piatti et al. (e.g. 2012), Piatti (e.g. 2012, 2015, and references therein), together with the whole data set for the aforementioned CTIO programme, which comprises 17 different LMC fields (see, Fig. 1) and used the MSCRED package in $\mathrm{IRAF}^{2}$. Point-spread function photometry was obtained by employing the DAOPHOT/ALLSTAR, DAOMATCH, and DAOMASTER suite of programs $^{3}$ (Stetson et al. 1990; Piatti et al. 2012; Piatti 2015). The photometric errors were computed as

\footnotetext{
1 http://www.noao.edu/sdm/archives.php

2 IRAF is distributed by the National Optical Astronomy Observatories, which is operated by the Association of Universities for Research in Astronomy, Inc., under contract with the National Science Foundation.

3 Provided kindly by Peter Stetson.
} 


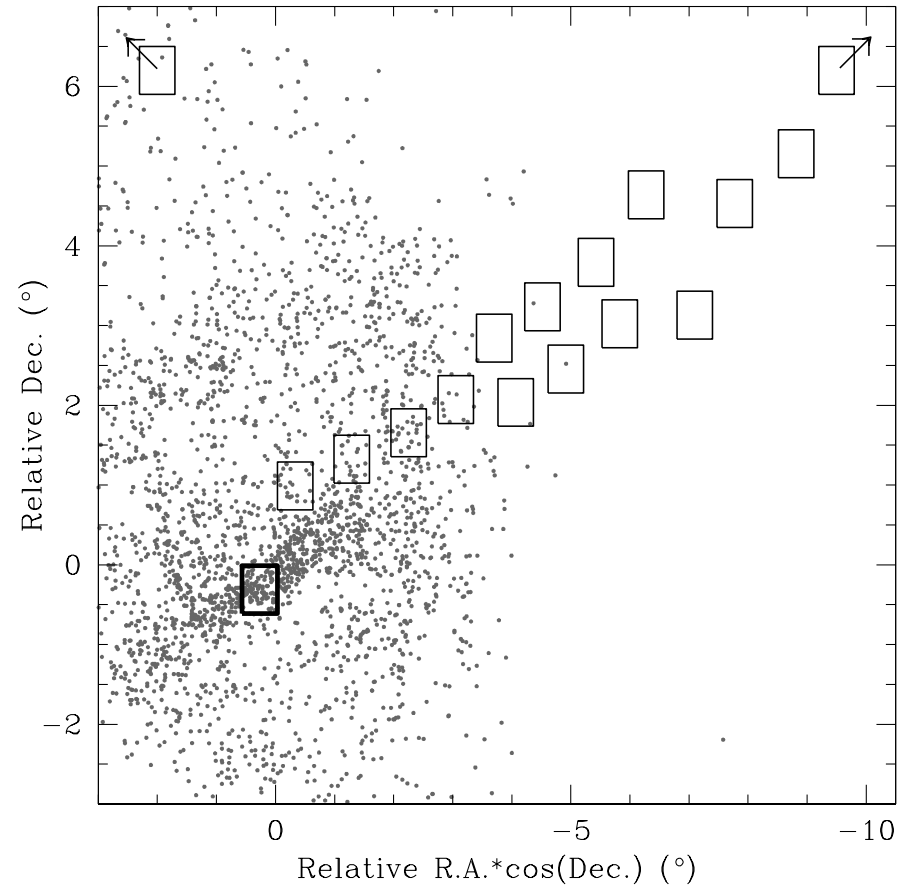

Fig. 1. Spatial location of the LMC star field studied here (thick black box), along with the remaining CTIO programme fields. Star clusters catalogued by Bica et al. (2008) are also drawn (dots) for comparison purposes.

described in Piatti \& Bastian (2016), Piatti \& Cole (2017). Figure 3 (top left panel) illustrates with error bars at the left margin the typical photometric errors. The 50 per cent completeness level is reached at $C \sim T_{1} \approx 20.0 \mathrm{mag}$ (see e.g. Piatti \& Cole 2017).

\section{Identification of star clusters}

We identified star clusters using an upgraded version of the procedure developed in Piatti et al. (2016) that has also been successfully used elsewhere (e.g. Piatti 2016, 2017). We particularly used a cut-off density of 1.5 times the local background dispersion above the mean background value. In our case, we generated a stellar density surface over the studied region from 902095 stars with positions and magnitudes measured in the two $C T_{1}$ filters. Here we finally obtained 73 star cluster candidates, all of them included in B08, except for a new cluster candidate centred at $(\mathrm{RA}$, Dec $)=(81.099754,-69.609673)(\mathrm{J} 2000.0)$.

We extracted from B08 every object with (RA, Dec) coordinates within the boundaries of the surveyed area in order to check whether the cluster search could pass over any catalogued cluster. We found that 39 catalogued clusters were not identified; one of them ([SL63] 443) because it falls on a Mosaic II image gap. The other 38 objects (see Table 1) could not be recognized when visually inspecting the $C$ and $T_{1}$ images, since the distribution of stars in their respective fields do not resemble that of an stellar aggregate. We consider them as probable non-genuine star clusters. The analysed crowded region shows high star field density variations that in addition to the particular spatial resolution used and magnitude limit reached by previous cataloguing works, could lead them to infer the existence of extended objects (sometimes not resolved). The catalogue of B08 includes objects discovered by the Optical Gravitational Lens Experiment (Udalski 2003, OGLE III), whose depth is of the order of 1.5 mag shallower than the Magellanic Cloud Photometric Survey (Zaritsky et al. 2004, MCPS), which in turn reaches
Table 1. Probable non-genuine objects in the catalogue of B08.

\begin{tabular}{ccc}
\hline \hline Cluster name & Cluster name & Cluster name \\
\hline BSDL 1340 & [HS66] 252 & OGLE-CL LMC 434 \\
BSDL 1353 & [HS66] 255 & OGLE-CL LMC 435 \\
BSDL 1522 & [HS66] 259 & OGLE-CL LMC 437 \\
BSDL 1540 & OGLE-CL LMC 375 & OGLE-CL LMC 439 \\
BSDL 1592 & OGLE-CL LMC 406 & OGLE-CL LMC 441 \\
BSDL 1597 & OGLE-CL LMC 410 & OGLE-CL LMC 443 \\
BSDL 1614 & OGLE-CL LMC 412 & OGLE-CL LMC 448 \\
BSDL 1636 & OGLE-CL LMC 414 & OGLE-CL LMC 455 \\
BSDL 1647 & OGLE-CL LMC 421 & OGLE-CL LMC 465 \\
BSDL 1680 & OGLE-CL LMC 425 & OGLE-CL LMC 466 \\
BSDL 1681 & OGLE-CL LMC 428 & OGLE-CL LMC 474 \\
BSDL 1768 & OGLE-CL LMC 430 & OGLE-CL LMC 475 \\
BSDL 1784 & OGLE-CL LMC 433 & \\
\hline & &
\end{tabular}

a limiting magnitude $V \sim 20$ mag (Noël et al. 2009). Our limiting magnitude is $T_{1} \approx 22.5 \mathrm{mag}$ (Piatti et al., in prep.). As an example, Fig. 2 compares an enlargement of the $R$ image centred on OGLE-CL LMC 414 to that obtained from the DSS Red one. The version of the figure with all objects listed in Table 1 is available in Appendix B. The 38 probable non-genuine physical systems represent $\sim 33$ per cent of all objects located within the analysed LMC bar field, catalogued by B08. This percentage is much higher than the percentages found by Piatti \& Bica (2012) and Piatti (2014b) for other Magellanic Cloud regions.

\section{Star cluster CMD cleaning}

We statistically suctracted field stars from the cluster CMDs by applying the procedure developed by Piatti \& Bica (2012) that has successfully been used elsewhere (e.g. Piatti 2014b; Piatti et al. 2015b,a; Piatti \& Bastian 2016, and references therein). Here we used four star-field CMDs constructed from stars within circles placed to the north, east, south, and west, adjacent to the cluster region, and with areas equal to the circular area (typically with radii 2-3 times the cluster radius) used for the cluster region. As a result, three objects (BSDL 1719, [HS66] 250, and [HS66] 291) - whose cleaned CMDs do not show any detectable trace of star cluster sequences - were discarded.

Figure 3 illustrates the performance of the cleaning procedure for OGLE-CLLMC 377. The 70 individual photometric catalogues for the confirmed clusters are provided in the online version of the journal. The columns of each catalogue successively list the star ID, the RA and Dec, the magnitude and error in $C$ and $T_{1}$, respectively, and the photometric membership probability $(P)$. The latter is encoded with numbers $1-4$ to represent probabilities of $25,50,75$, and 100 per cent, respectively.

According to Piatti \& Geisler (2013, see their Fig. 6), LMC star clusters mostly expand the age range $\log \left(t \mathrm{yr}^{-1}\right) \lesssim 9.40$, with the exception of ESO 121-SC-03 $\left(\log \left(t \mathrm{yr}^{-1}\right) \sim 9.92\right)$ and 15 old globular clusters $\left(\log \left(t \mathrm{yr}^{-1}\right) \sim 10.1\right)$. Young star clusters are distinguished in the CMDs by their bright MSs, while intermediateage clusters $\left(9<\log \left(t \mathrm{yr}^{-1}\right)<9.45\right)$ have MS turnoffs (TOs) that decrease in brightness as they become older. A typical $2.5 \mathrm{Gyr}$ old LMC cluster $\left(\log \left(t \mathrm{yr}^{-1}\right)=9.4\right)$ has its MS TO at $T_{1} \sim$ 20.5 mag. By assuming a depth of the LMC disc of $(3.44 \pm$ 1.16) kpc (Subramanian \& Subramaniam 2009) and that such a cluster is located behind the LMC, its MS TO would result $\Delta T_{1} \lesssim$ 0.3 mag fainter. This means that the faintest cluster MS TO stars typically seen in the LMC are brighter than $T_{1} \approx 21.0$ mag. This magnitude is even brighter that our limiting magnitude, so that 

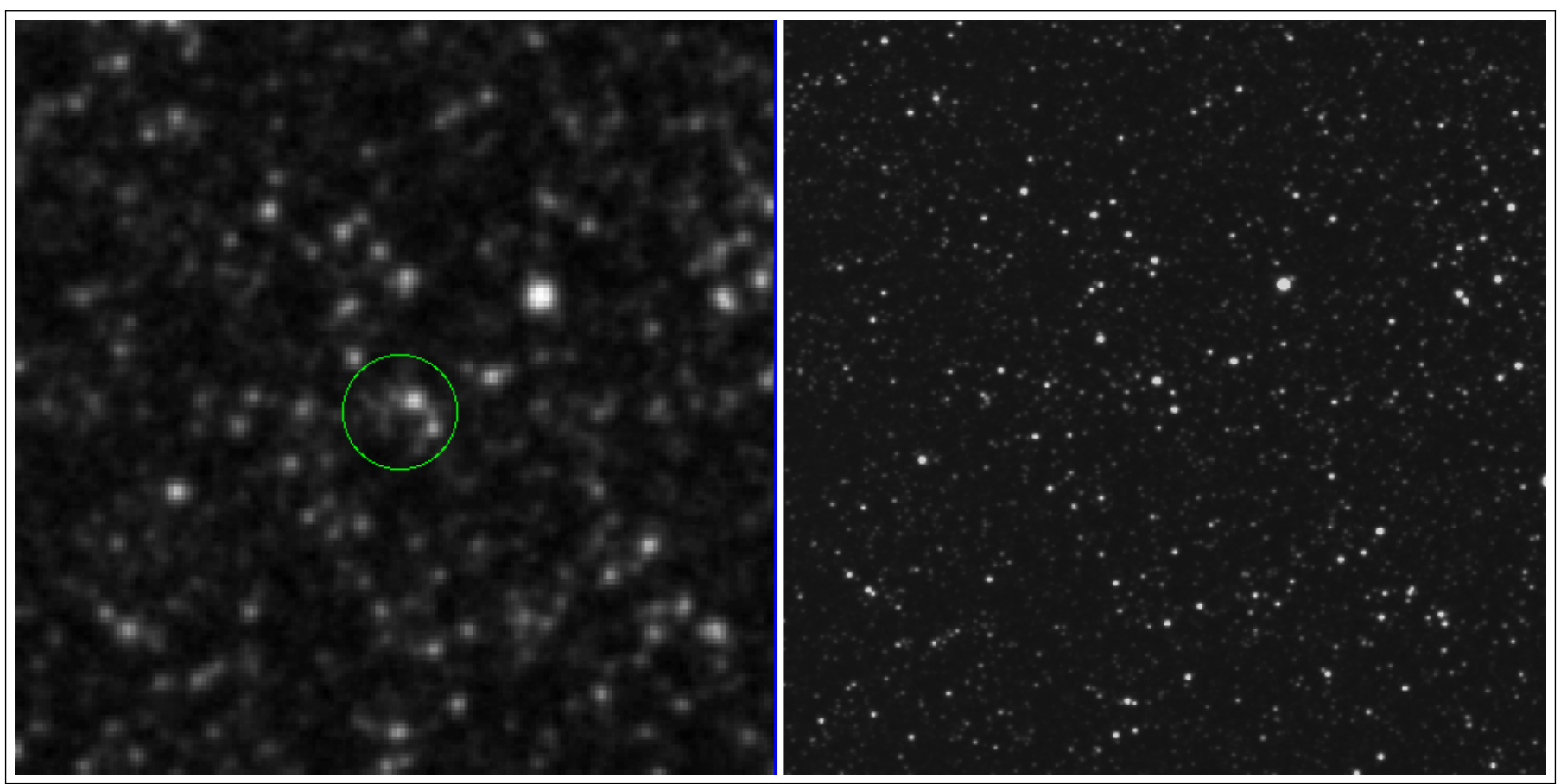

Fig. 2. $3 \times 3 \operatorname{arcmin}^{2}$ DSS Red (left) and $R$ (right) images centred on OGLE-CL LMC 414, previously catalogued as a cluster and not recovered as such in the present work. North is up and East to the left. The green circle illustrates the angular dimension given in B08.

we were able to detect any star cluster (with stars from its brightest limit down to its MS TO) located in the surveyed field.

\section{Star cluster ages}

We estimated the ages of the confirmed star clusters using their CMDs built from stars with membership probabilities higher than 50 per cent and matching them with the theoretical isochrones of Bressan et al. (2012). In performing this task, we dealt with their reddenings, distances, and metallicities. As for the cluster metallicies, we adopted a value of $[\mathrm{Fe} / \mathrm{H}]=-0.4 \mathrm{dex}$ for all of them (Piatti \& Geisler 2013). Consequently, if we were allow the metallicity to vary, we would not be able to see any meaningful difference along the cluster MSs because of the dispersion of the stars. We made one exception in the employment of isochrones for the old globular cluster NGC 1939, for which we adopted $[\mathrm{Fe} / \mathrm{H}]=-2.0$ dex.

We took advantage of the Magellanic Clouds (MCs) extinction values based on red clump (RC) and RR Lyrae stellar photometry provided by the OGLE III collaboration, as described in Haschke et al. (2011), to estimate $E(V-I)$ colour excesses. We recall that they found very low reddenings in the LMC bar region. In matching isochrones, we started by adopting those $E(V-I)$ values, combined with the equations $E(V-I) / E(B-V)=1.25, A_{V} / E(B-V)=3.1$ (Cardelli et al. 1989); $E\left(C-T_{1}\right) / E(B-V)=1.97$ and $A_{T_{1}} / E(B-V)=2.62$ (Geisler 1996). We note that considering the LMC disc depth, the difference in distance modulus could be as large as $\Delta(m-M)_{o} \sim$ $0.3 \mathrm{mag}$, which is of the order of the uncertainties while adjusting isochrones to the cluster CMDs in magnitude (nearly twice as large as the size of the plotting symbols in Fig. 3), so that our simple assumption of adopting the same distance of all clusters should not affect the results.

Table 2 lists the derived $E(V-I)$ colour excesses and ages, while Fig. 3 (bottom left panel) illustrates the performance of the isochrone matching. We estimated an upper value for our age uncertainties of $\Delta \log \left(t \mathrm{yr}^{-1}\right)= \pm 0.10$.

\section{Star cluster analysis}

Only few clusters in our sample have previously been studied from resolved stellar photometry. Mackey \& Gilmore (2004) presented HST data that resulted in high-accuracy CMDs for NGC 1938 and NGC 1939. Our $C T_{1}$ photometry confirms their results for the old globular cluster NGC $1939\left(\log \left(t \mathrm{yr}^{-1}\right)=10.1\right.$, $[\mathrm{Fe} / \mathrm{H}]=-2.0 \mathrm{dex})$ and gives an age slightly older than and within the quoted uncertainties of the value derived by them $\left(\log \left(t \mathrm{yr}^{-1}\right) \sim 8.6\right)$ for NGC 1938. Dieball \& Grebel (2000) obtained Gunn $g, i$ photometry at the ESO/MPI $2.2 \mathrm{~m}$ telescope (La Silla) for the triple system NGC 1969, 1971, and 1972, and derived ages of $\log \left(t \mathrm{yr}^{-1}\right)=7.8,7.8$, and 7.6 with a typical error of $\sigma\left(\log \left(t \mathrm{yr}^{-1}\right)\right)= \pm 0.1$, respectively, from the matching of theoretical isochrones. These values are younger than those derived here, and could be mostly affected by star field contamination; particularly of bright field stars assumed to be cluster stars (see their Fig. 7). We note that they did not perform any decontamination of field stars in their CMDs.

The VISTA ${ }^{4}$ near-infrared $Y J K_{s}$ survey of the MCs system (Cioni et al. 2011, VMC) has also imaged three clusters of our sample, namely KMK88 55, OGLE-CL LMC 451 (三[HS66] 282), and OGLE-CL LMC 469 (三[HS66] 295). They were studied by Piatti et al. (2014) from CMDs built using PSF photometry on homogenised deep tile images (Rubele et al. 2012). KMK88 55 was found to be a cluster of $\log \left(t \mathrm{yr}^{-1}\right) \sim 8.5$, while OGLE-CL LMC 451 and OGLE-CL LMC 469 were classified as probable non-genuine star clusters. The older age derived for KMK8855 is affected by the lack of measurements of blue cluster stars, while the assessment of the physical reality of OGLE-CL LMC 451 and OGLE-CL LMC 469 is based

\footnotetext{
4 Visible and Infrared Survey Telescope for Astronomy.
} 


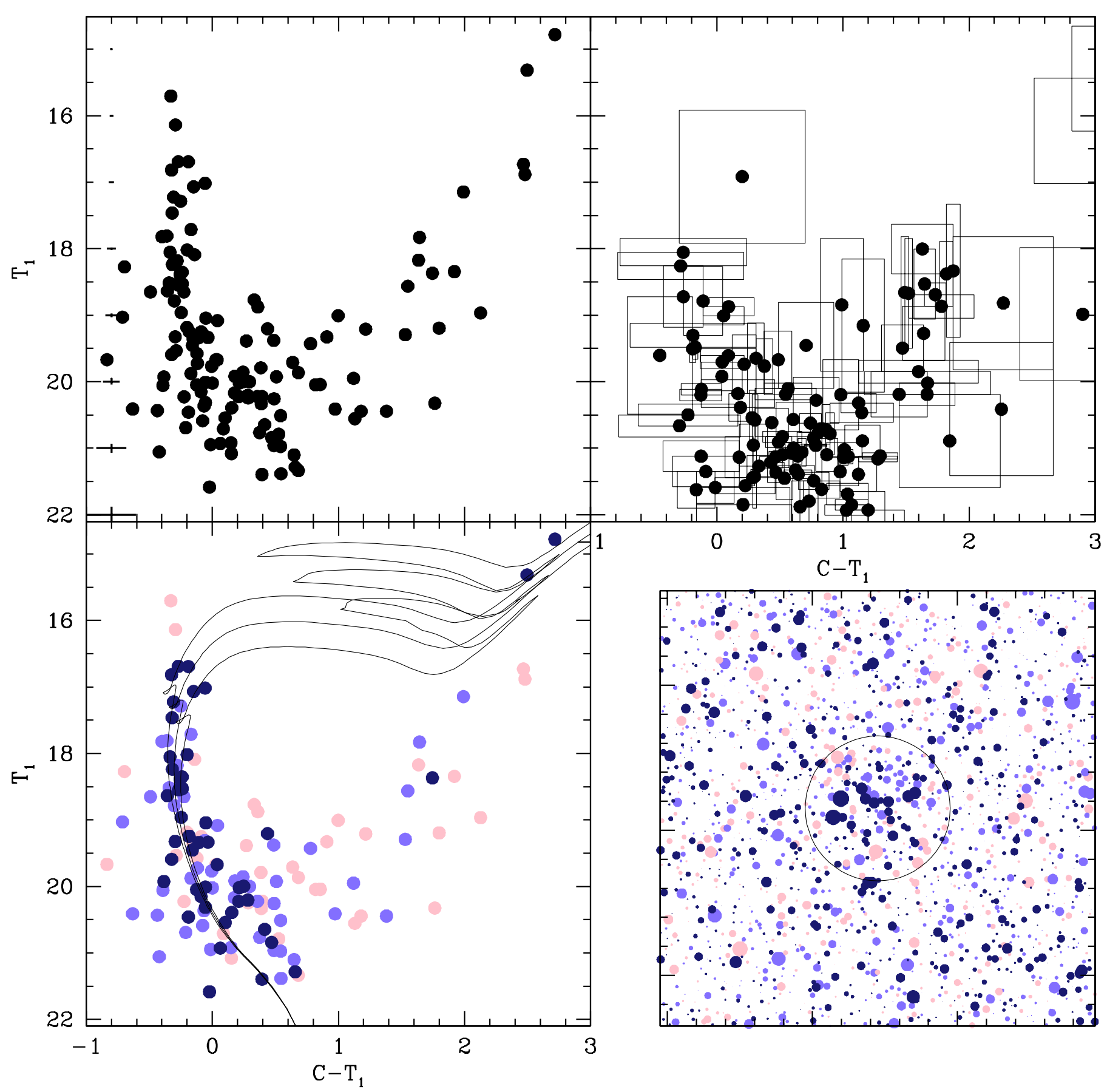

Fig. 3. CMDs for stars in the field of OGLE-CL LMC 377: the observed CMD composed of the stars distributed within the cluster radius, with typical photometric errors represented with error bars at the left margin (top left-hand panel); a field CMD for a circular area equal to that of the cluster with the respective sample of produced boxes used in the cleaning procedure (top right-hand panel); the cleaned cluster CMD (bottom left). Colour-scaled symbols represent stars with membership probability of $P \leq 25 \%$ (pink), $P=50 \%$ (light blue), and $P \geq 75 \%$ (dark blue). Three isochrones from Bressan et al. (2012) for $\log \left(t \mathrm{yr}^{-1}\right)=8.1,8.2$, and 8.3 and $Z=0.006$ are also superimposed. The schematic diagram centred on the cluster is shown in the bottom right-hand panel. The black circle represents the adopted cluster radius. Symbols are as in the bottom left-hand panel, with sizes proportional to the stellar brightnesses. North is up; east is to the left. The actual images are shown in Fig. A.1.

on a shallower VMC $K_{\mathrm{s}}$ limiting magnitude. We show in Fig. 4 the cleaned CMDs constructed by Piatti et al. (2014) compared to ours.

Most of the remaining clusters in our sample, as well as those probable non-genuine clusters of Table 1 , do have only age estimates on the basis of integrated colours (Pandey et al. 2010; Popescu et al. 2012). However, Asa'd et al. (2013) showed that unresolved methods (integrated broad-band colour photometry) poorly match the ages of LMC clusters derived from resolved stellar photometry (CMD). Piatti (2014a) also found results similar to those of Asa'd et al. (2013) when integrated spectroscopy was used to estimate cluster ages.

The star cluster frequency $(\mathrm{CF})$ - the number of clusters per time unit as a function of age - is a straightforward way to compare the cluster formation activity in different epochs of the galaxy lifetime. In the case of the LMC, it has been built 
Table 2. Fundamental properties of the star cluster sample.

\begin{tabular}{lcclcc}
\hline \hline Cluster name & $E(V-I)^{a}$ & $\log \left(t \mathrm{yr}^{-1}\right)$ & Cluster name & $E(V-I)^{a}$ & $\log \left(t \mathrm{yr}^{-1}\right)$ \\
\hline BRHT 50a & 0.04 & 8.15 & NGC 1959 & 0.04 & 8.70 \\
BSDL 1291 & 0.04 & 8.20 & NGC 1958 & 0.05 & 8.60 \\
BSDL 1299 & 0.04 & 8.40 & NGC 1969 & 0.04 & 8.30 \\
BSDL 1335 & 0.11 & 8.00 & NGC 1971 & 0.03 & 8.20 \\
BSDL 1367 & 0.03 & 8.40 & NGC 1972 & 0.03 & 8.40 \\
BSDL 1381 & 0.03 & 8.20 & OGLE-CLLMC 369 & 0.04 & 8.55 \\
BSDL 1480 & $(0.20)$ & 7.30 & OGLE-CLLMC 376 & $(0.20)$ & 7.30 \\
BSDL 1491 & $(0.15)$ & 8.30 & OGLE-CL LMC 377 & 0.08 & 8.20 \\
BSDL 1511 & 0.07 & 8.20 & OGLE-CLLMC 396 & 0.08 & 8.55 \\
BSDL 1516 & 0.07 & 8.15 & OGLE-CLLMC 398 & $(0.30)$ & 8.20 \\
BSDL 1576 & 0.04 & 8.60 & OGLE-CL LMC 400 & $(0.25)$ & 8.00 \\
BSDL 1601 & 0.05 & 8.20 & OGLE-CLLMC 402 & $(0.20)$ & 8.15 \\
BSDL 1608 & 0.04 & 8.10 & OGLE-CLLMC 403 & $(0.20)$ & 8.25 \\
BSDL 1707 & 0.06 & 9.00 & OGLE-CL LMC 407 & 0.04 & 8.70 \\
BSDL 1712 & $(0.10)$ & 8.00 & OGLE-CLLMC 415 & $(0.15)$ & 8.15 \\
BSDL 1723 & 0.04 & 8.35 & OGLE-CL LMC 416 & 0.09 & 8.20 \\
BSDL 1772 & 0.04 & 8.50 & OGLE-CL LMC 418 & $(0.15)$ & 8.55 \\
BSDL 1778 & 0.03 & 8.75 & OGLE-CLLMC 419 & 0.12 & 8.05 \\
BSDL 1785 & 0.04 & 8.35 & OGLE-CL LMC 420 & 0.04 & 8.70 \\
H88 283 & 0.03 & 8.55 & OGLE-CL LMC 429 & 0.02 & 8.40 \\
H88 295 & 0.02 & 8.75 & OGLE-CLLMC 431 & 0.04 & 8.05 \\
[HS66] 251 & 0.02 & 8.55 & OGLE-CL LMC 438 & $(0.10)$ & 8.65 \\
KMK88 48 & 0.04 & 8.90 & OGLE-CL LMC 442 & 0.04 & 9.00 \\
KMK88 49 & 0.09 & 8.70 & OGLE-CLLMC 447 & 0.02 & 8.40 \\
KMK88 50 & 0.09 & 8.75 & OGLE-CL LMC 451 & 0.07 & 8.80 \\
KMK88 51 & 0.12 & 8.30 & OGLE-CL LMC 456 & 0.04 & 8.60 \\
KMK88 52 & $(0.15)$ & 8.05 & OGLE-CLLMC 462 & 0.05 & 8.70 \\
KMK88 55 & 0.08 & 8.20 & OGLE-CL LMC 463 & 0.06 & 8.60 \\
KMK88 56 & $(0.15)$ & 8.45 & OGLE-CL LMC 467 & 0.05 & 8.25 \\
KMK88 57 & $(0.20)$ & 8.55 & OGLE-CL LMC 468 & 0.06 & 8.20 \\
newcls & 0.04 & 8.10 & OGLE-CL LMC 469 & 0.07 & 8.70 \\
NGC 1926 & 0.03 & 8.35 & OGLE-CL LMC 472 & 0.05 & 7.60 \\
NGC 1938 & 0.07 & 8.70 & OGLE-CL LMC 478 & 0.05 & 8.65 \\
NGC 1939 & 0.07 & 10.10 & OGLE-CLLMC 479 & 0.06 & 8.20 \\
NGC 1950 & 0.04 & 8.70 & [SL63]436 & 0.04 & 8.60 \\
\hline
\end{tabular}

Notes. ${ }^{(a)} E(V-I)$ values in parentheses are slightly higher than those from Haschke et al. (2011) to better match the isochrones. Nevertheless, they are within the dispersion given for the OGLE III $E(V-I)$ colour excesses.

for different regions and been found to vary from one place to another (Piatti 2014b,c, and references therein). Moreover, variations within the LMC bar have also been found (e.g. Piatti et al. 2015a). Therefore, aiming at tracing the intrinsic cluster formation history in the surveyed area, we built its CF from the ages estimated for the 70 studied star clusters.

Instead of constructing an age histogram, we assigned to each cluster a Gaussian distribution centred on the mean cluster age and with FWHM twice as large as the age uncertainty. The result of summing the contribution of all Gaussian distributions is depicted in Fig. 5. For comparison purposes, the CF was normalized to the total number of clusters. As can be seen, the main star cluster formation activity has taken place during the period $\log \left(t \mathrm{yr}^{-1}\right) \sim 8.0-9.0$, suggesting that either clusters in this bar region have been formed relatively recently, or any cluster older than $\log \left(t \mathrm{yr}^{-1}\right) \sim 9$ has been disrupted. The only exception is the old globular cluster NGC 1939, which could also be an outer disc cluster projected on the LMC bar (Sharma et al. 2010). Nevertheless, since Piatti (2014c) found for the whole LMC bar that there has been cluster formation activity from $\log \left(t \mathrm{yr}^{-1}\right) \sim$ 9.4 , we conclude that this part of the bar is on average a relatively particular younger one. The cluster formation during the last 100 Myr shows some short isolated periods of true activity.
The derived $\mathrm{CF}$ was finally compared with the $\mathrm{CF}$ obtained from the star formation rate (SFR) derived by Smecker-Hane et al. (2002) using HST observations. We used their SFR and cluster masses from $\log \left(M_{\mathrm{cl}}\left[M_{\odot}\right]\right)=2.2$ to $\log \left(M_{\mathrm{cl}}\left[M_{\odot}\right]\right)=5.0$ (de Grijs \& Goodwin 2008; Glatt et al. 2011). Figure 5 shows the resulting recovered $C F$ drawn with a solid line. The observed and recovered CFs are clearly different for several reasons. On the one hand, the recovered $\mathrm{CF}$ shows star formation activity where there is no cluster $\left(\log \left(t \mathrm{yr}^{-1}\right) \gtrsim\right.$ 9.0). At first glance, this could be somewhat surprising when we take into account the common notion that most of the stars more massive than $0.50 .5 M_{\odot}$ may form in clusters, so that a significant fraction of field stellar populations originate from disrupted clusters (e.g. Lada \& Lada 2003). However, the LMC exhibits a well-known gap in the cluster age distribution between $\log \left(t \mathrm{yr}^{-1}\right) \sim 9.5-10.1$, while the age distribution of the field stellar population appears more continuous (Piatti \& Geisler 2013). Furthermore, numerous authors have asserted that the LMC's field star and star cluster formation histories are significantly different (e.g. Olszewski et al. 1996; Geha et al. 1998; Sarajedini 1998, and references therein).

On the other hand, the observed CF shows a noticeable excess with respect to the recovered $\mathrm{CF}$ for ages younger than 

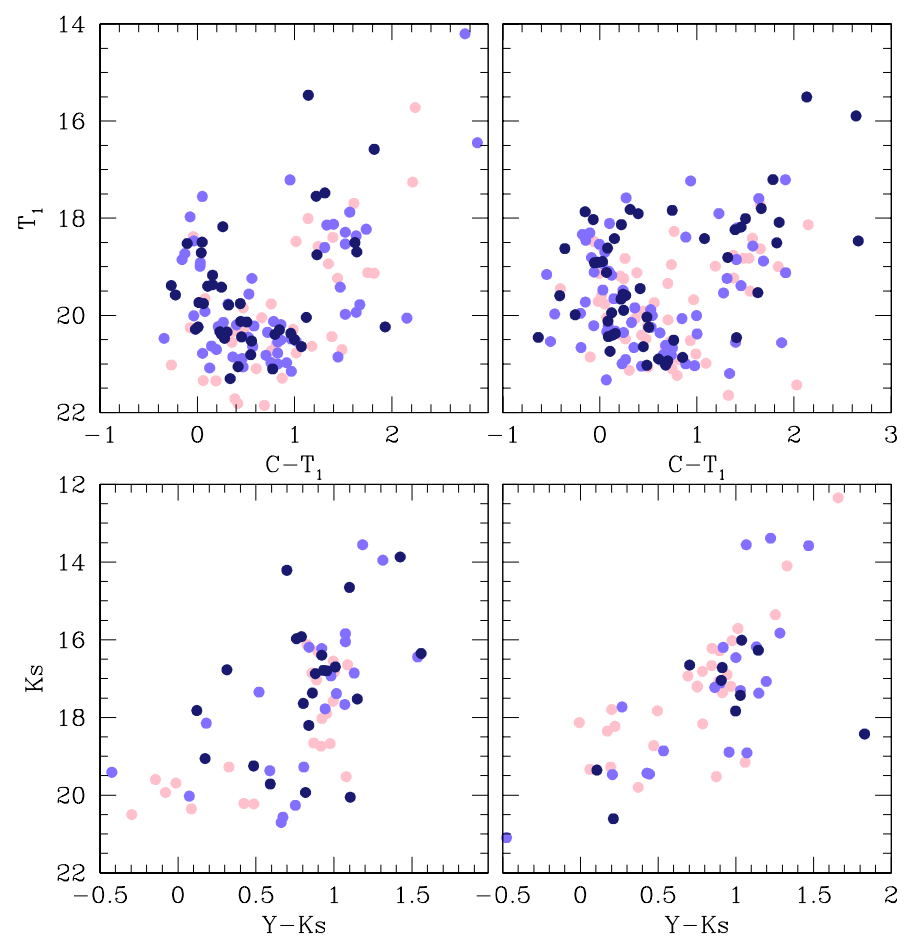

Fig. 4. Cleaned CMDs for OGLE-CLLMC 451 (left) and OGLECLLMC 469 (right) in the Washington $C T_{1}$ (top) and $Y K_{\mathrm{s}}$ (bottom) filters.

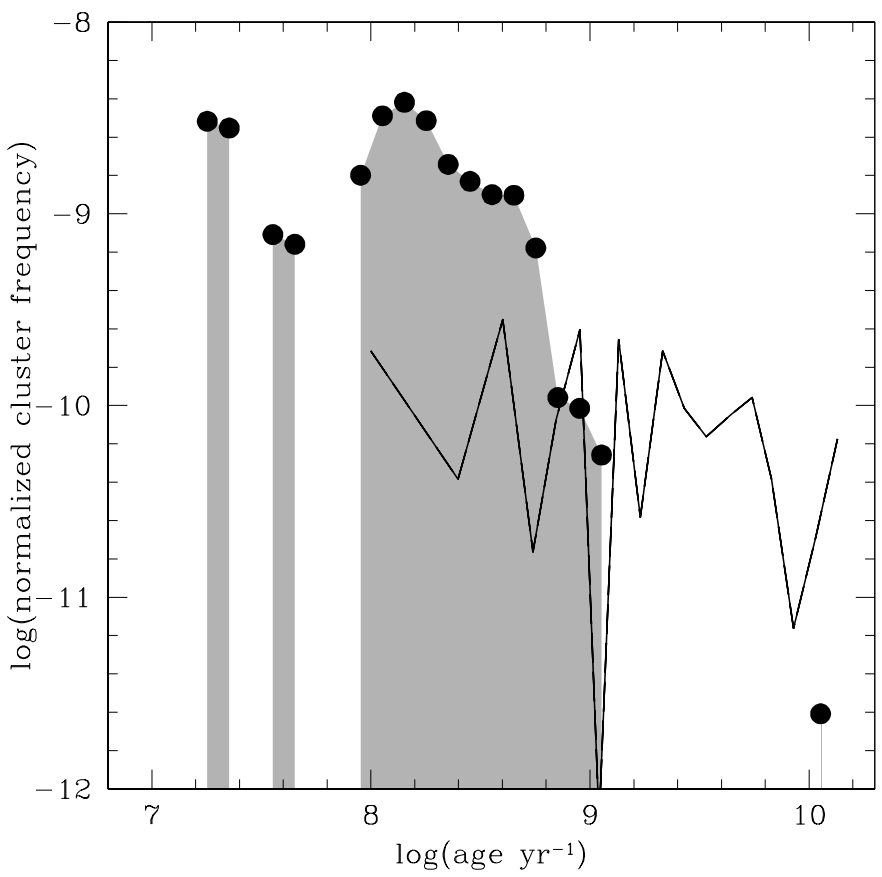

Fig. 5. CF of the surveyed region in the LMC bar (filled circles). The grey areas highlight the periods of star cluster formation activity, while the solid line represents the CF recovered from the corresponding SFR obtained by Smecker-Hane et al. (2002).

$\log \left(t \mathrm{yr}^{-1}\right) \sim 9.0$. Even though the recovered CF requires additional refinements, the observed disparities between the cluster and field star age distributions seem to offer evidence in support of a decoupling between star cluster and field star formation. These results provide some clues for a better approach in the study of the field stars origin and its link to cluster disruption and environmental conditions.

\section{Conclusions}

In this work we analyse CMDs of star clusters located in the South-Eastern half of the LMC bar from a Washington $C T_{1}$ photometric data set.

We performed a procedure for the star cluster search which consists in using Gaussian and tophat KDEs with a bandwidth of 0.4 arcmin, and detected 73 star cluster candidates. We did not detect other 38 previously catalogued clusters, which could not be recognized when visually inspecting the $C$ and $T_{1}$ images either. The distribution of stars in their respective fields do not resemble that of an stellar aggregate. We consider them as probable non-genuine star clusters. The 38 probable non-genuine physical systems represent $\sim 33$ per cent of all catalogued objects located within the analysed LMC bar field.

The CMDs of the star cluster candidates were statistically cleaned from field star contamination. Three objects, whose CMDs do not show any detectable trace of star cluster sequences, were discarded. The confirmed clusters comprises a complete sample, since we were able to detect any star cluster with stars from its brightest limit down to its MS TO located in the surveyed field. From matching theoretical isochrones to the cleaned cluster CMDs we estimated ages taking into account the LMC mean distance modulus, the present day metallicity and the individual star cluster colour excesses. As far as we are aware, these are the first age estimates based on resolved stellar photometry for most of the studied 70 clusters. The derived ages are in the age range $7.2<\log \left(t \mathrm{yr}^{-1}\right)<9.1$, in addition to the old globular cluster NGC 1939.

Finally, we built the CF aiming at tracing the intrinsic cluster formation history of the surveyed area. We found that the major star cluster formation activity has taken place during the period $\log \left(t \mathrm{yr}^{-1}\right) \sim 8.0-9.0$, which results in average relatively younger than the whole formation period of the LMC bar. Since $\sim 100$ Myr ago, clusters have been formed during few bursting formation events. When comparing the observed CF to that recovered from the SFR derived by Smecker-Hane et al. (2002) we found noticeable differences. We conclude that they are evidence of field star and star cluster formation histories are significantly different.

Acknowledgements. We thank the referee for his thorough reading of the manuscript and timely suggestions to improve it.

\section{References}

Asa'd, R. S., Hanson, M. M., \& Ahumada, A. V. 2013, PASP, 125, 1304 Bica, E., Bonatto, C., Dutra, C. M., \& Santos, J. F. C. 2008, MNRAS, 389, 678 Bressan, A., Marigo, P., Girardi, L., et al. 2012, MNRAS, 427, 127 Cardelli, J. A., Clayton, G. C., \& Mathis, J. S. 1989, ApJ, 345, 245

Cioni, M.-R. L., Clementini, G., Girardi, L., et al. 2011, A\&A, 527, A116 de Grijs, R., \& Goodwin, S. P. 2008, MNRAS, 383, 1000 Dieball, A., \& Grebel, E. K. 2000, A\&A, 358, 897 Geha, M. C., Holtzman, J. A., Mould, J. R., et al. 1998, AJ, 115, 1045 Geisler, D. 1996, AJ, 111, 480

Glatt, K., Grebel, E. K., Jordi, K., et al. 2011, AJ, 142, 36 Haschke, R., Grebel, E. K., \& Duffau, S. 2011, AJ, 141, 158 Lada, C. J., \& Lada, E. A. 2003, ARA\&A, 41, 57

Mackey, A. D., \& Gilmore, G. F. 2004, MNRAS, 352, 153

Nayak, P. K., Subramaniam, A., Choudhury, S., Indu, G., \& Sagar, R. 2016, MNRAS, 463, 1446 
A. E. Piatti: LMC bar star clusters

Noël, N. E. D., Aparicio, A., Gallart, C., et al. 2009, ApJ, 705, 1260 Olszewski, E. W., Suntzeff, N. B., \& Mateo, M. 1996, ARA\&A, 34, 511 Pandey, A. K., Sandhu, T. S., Sagar, R., \& Battinelli, P. 2010, MNRAS, 403, 1491

Piatti, A. E. 2012, MNRAS, 422, 1109

Piatti, A. E. 2014a, MNRAS, 445, 2302

Piatti, A. E. 2014b, MNRAS, 440, 3091

Piatti, A. E. 2014c, MNRAS, 437, 1646

Piatti, A. E. 2015, MNRAS, 451, 3219

Piatti, A. E. 2016, MNRAS, 459, L61

Piatti, A. E. 2017, ApJ, 834, L14

Piatti, A. E., \& Bastian, N. 2016, A\&A, 590, A50

Piatti, A. E., \& Bica, E. 2012, MNRAS, 425, 3085

Piatti, A. E., \& Cole, A. 2017, MNRAS, 470, L77

Piatti, A. E., \& Geisler, D. 2013, AJ, 145, 17

Piatti, A. E., Geisler, D., \& Mateluna, R. 2012, AJ, 144, 100
Piatti, A. E., Guandalini, R., Ivanov, V. D., et al. 2014, A\&A, 570, A74 Piatti, A. E., de Grijs, R., Ripepi, V., et al. 2015a, MNRAS, 454, 839 Piatti, A. E., de Grijs, R., Rubele, S., et al. 2015b, MNRAS, 450, 552 Piatti, A. E., Ivanov, V. D., Rubele, S., et al. 2016, MNRAS, 460, 383 Popescu, B., Hanson, M. M., \& Elmegreen, B. G. 2012, ApJ, 751, 122 Rubele, S., Kerber, L., Girardi, L., et al. 2012, A\&A, 537, A106 Sarajedini, A. 1998, AJ, 116, 738

Sharma, S., Borissova, J., Kurtev, R., Ivanov, V. D., \& Geisler, D. 2010, AJ, 139, 878

Smecker-Hane, T. A., Cole, A. A., Gallagher, III, J. S., \& Stetson, P. B. 2002, ApJ, 572, 1083

Stetson, P. B., Davis, L. E., \& Crabtree, D. R. 1990, in CCDs in astronomy, ed. G. H. Jacoby, ASP Conf. Ser., 8, 289

Subramanian, S., \& Subramaniam, A. 2009, A\&A, 496, 399

Udalski, A. 2003, Acta Astron., 53, 291

Zaritsky, D., Harris, J., Thompson, I. B., \& Grebel, E. K. 2004, AJ, 128, 1606 


\section{Appendix A: OGLE-CL LMC 377 images}

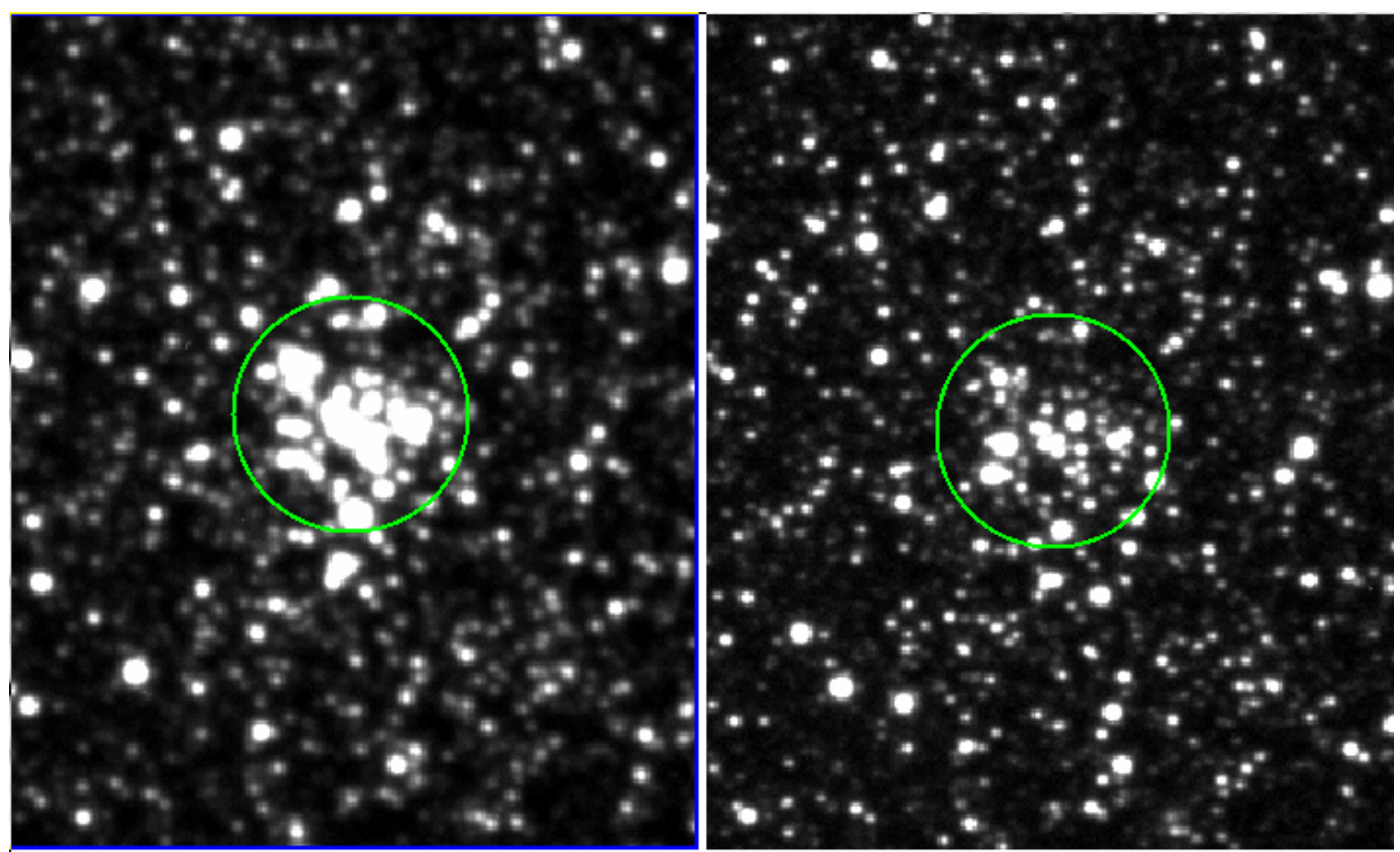

Fig. A.1. $C$ (left) and $R$ (right) images centred on OGLE-CL LMC 377. The circles are as in Fig. 3. 
Appendix B: Figure 2 (full version)

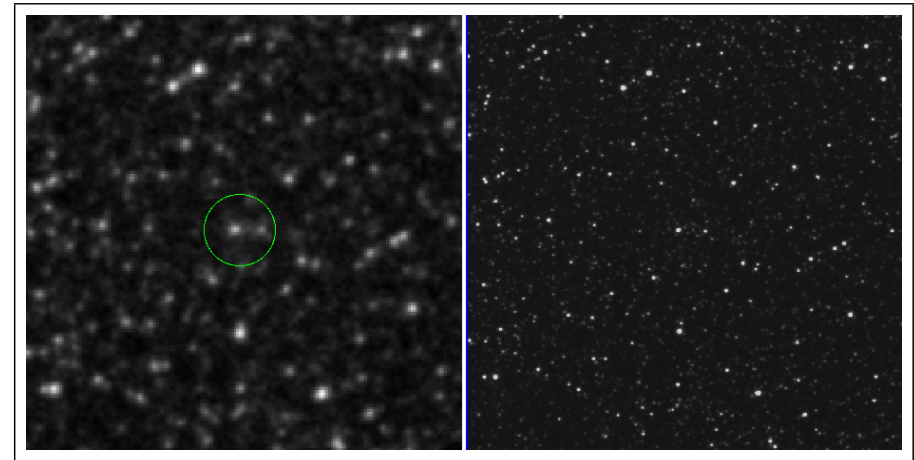

BSDL 1340
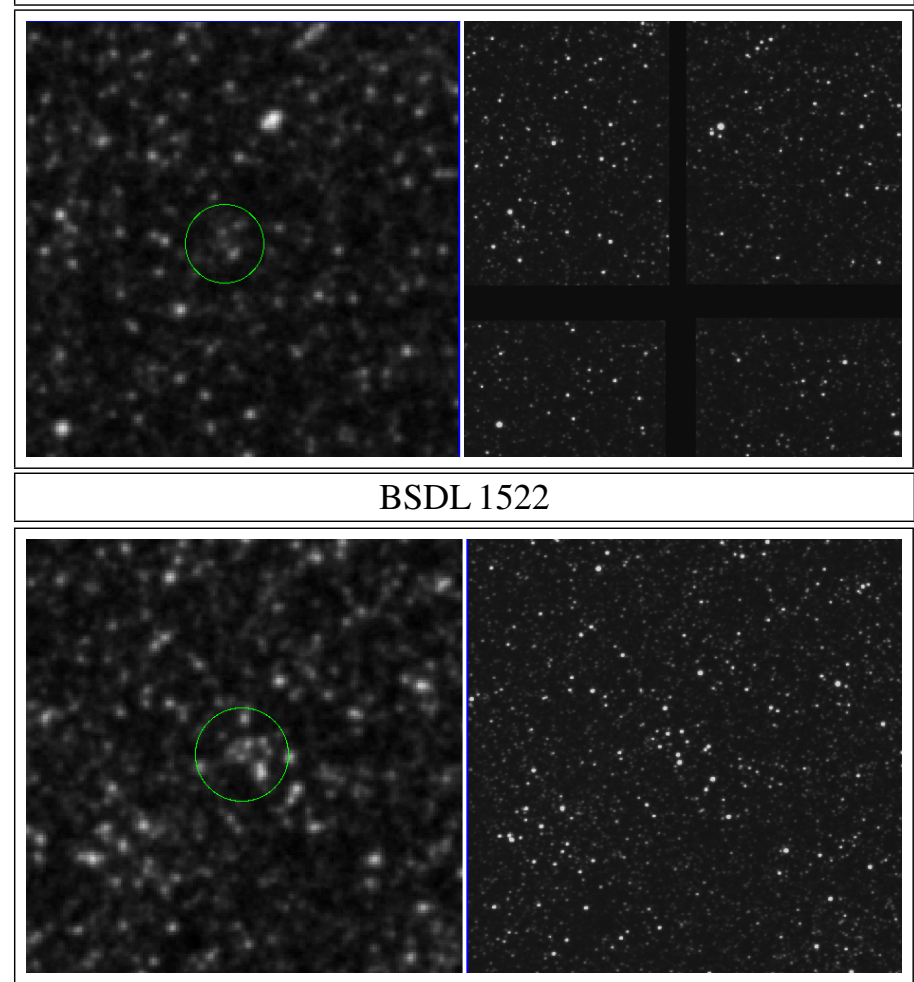

BSDL 1592

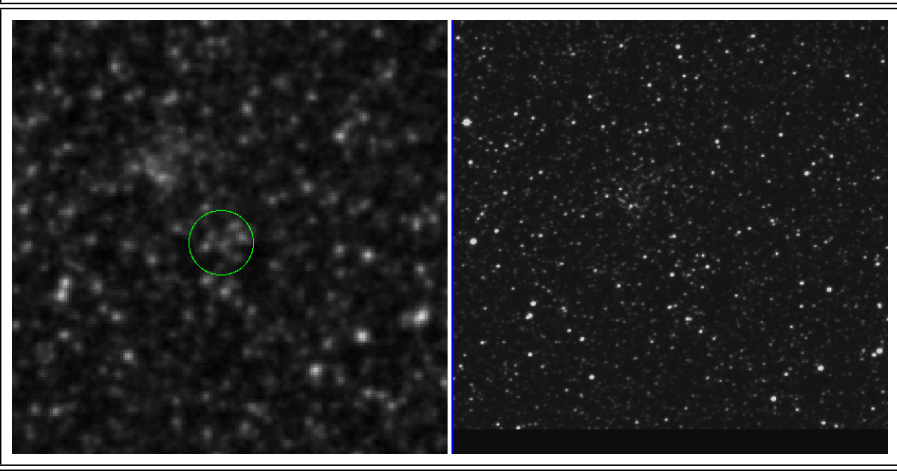

BSDL 1614

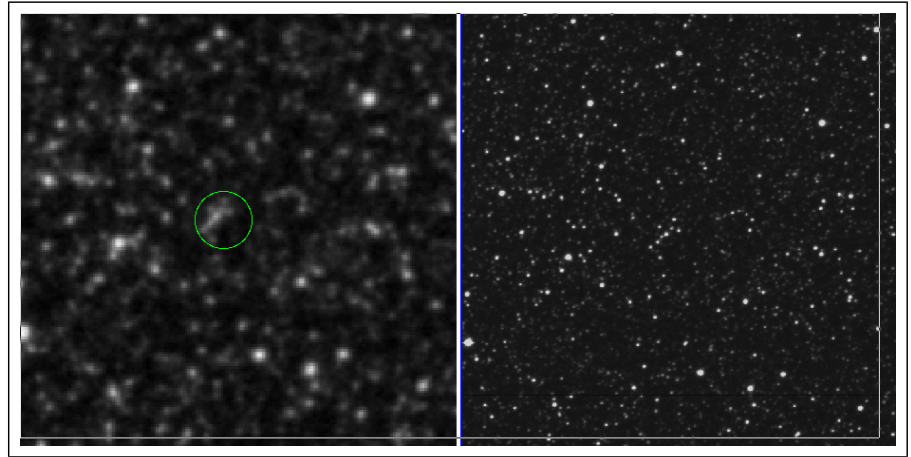

BSDL 1353

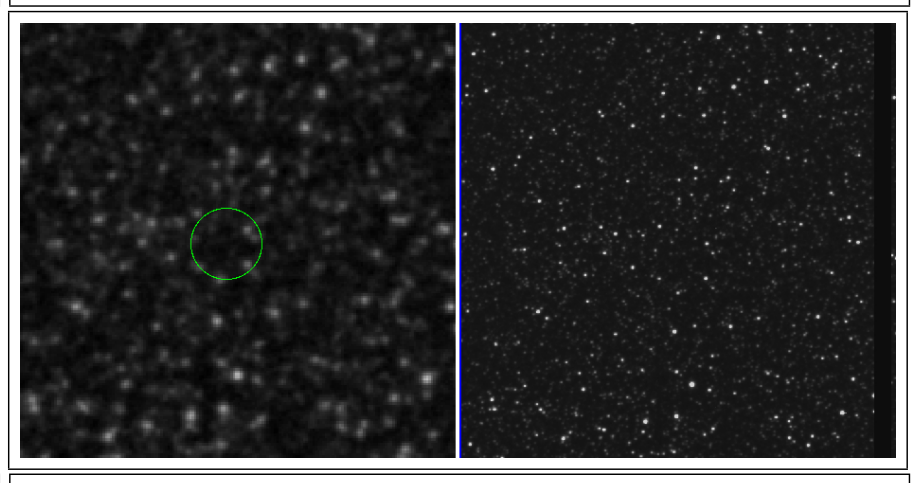

BSDL 1540
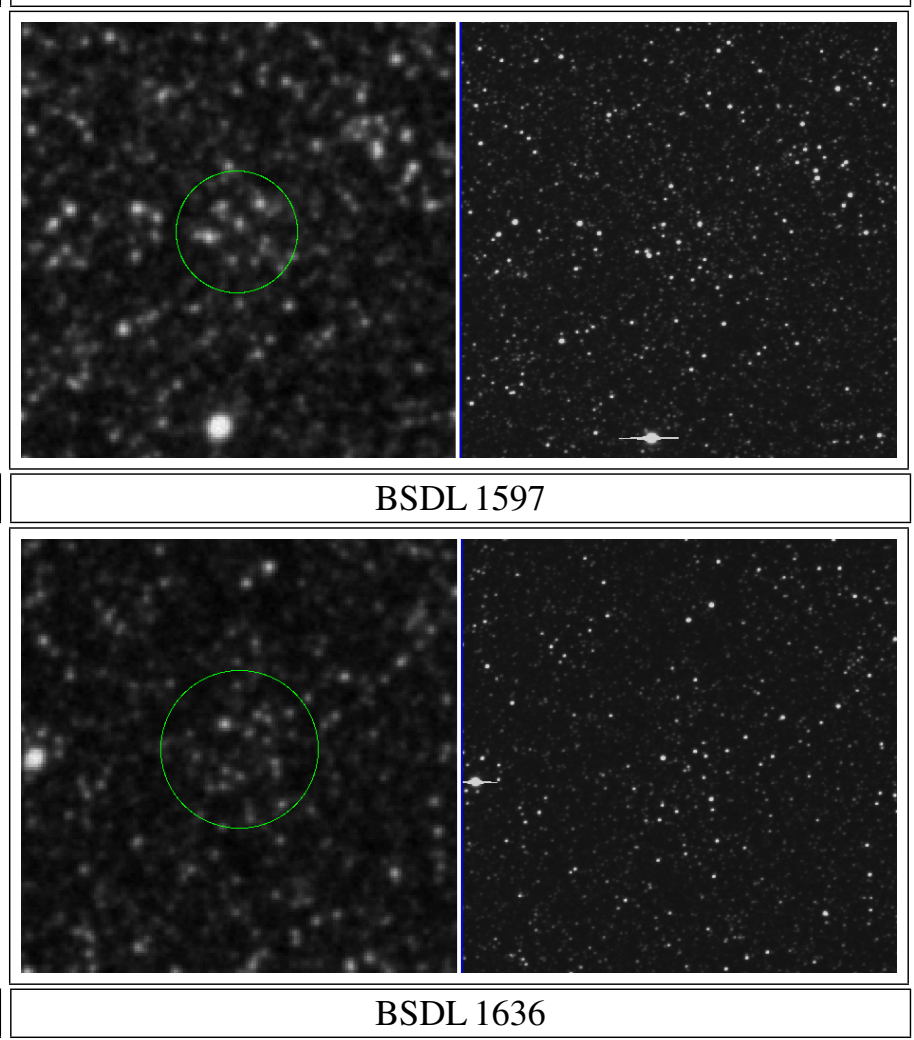

Fig. B.1. $3 \times 3 \operatorname{arcmin}^{2}$ DSS Red (left) and $R$ (right) images centred on objects catalogued by B08 (see text for details). North is up and East to the left. The green circle illustrates the angular dimension given in B08. 


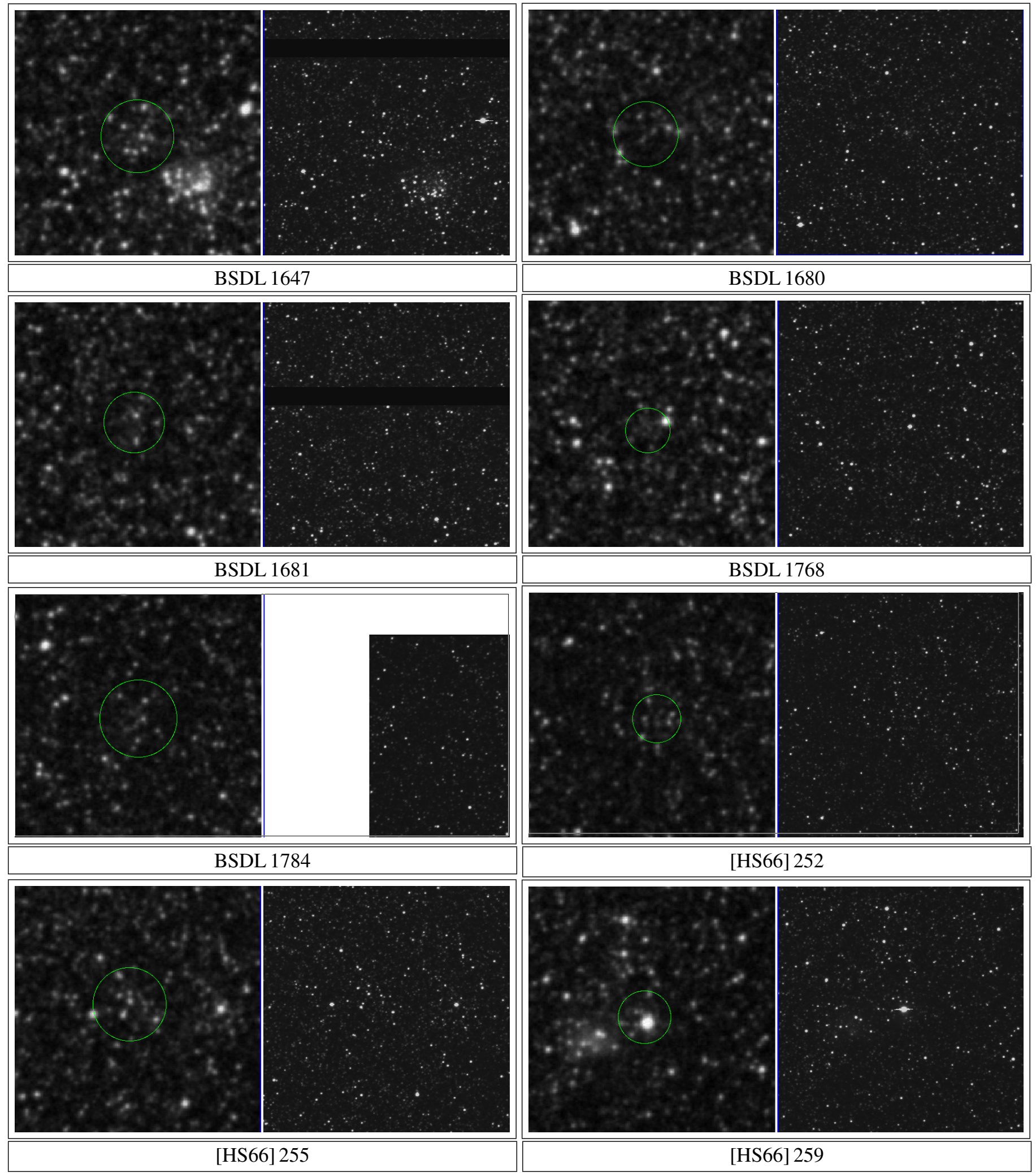

Fig. B.1. continued. 
A. E. Piatti: LMC bar star clusters
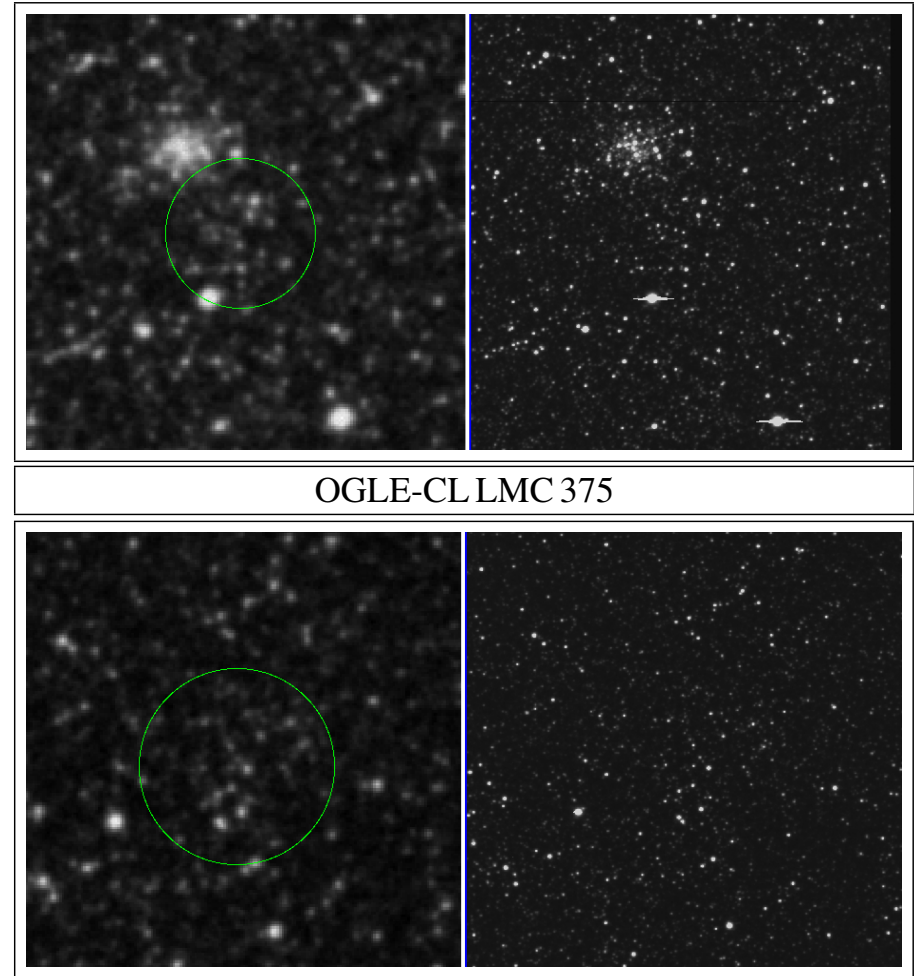

OGLE-CL LMC 410

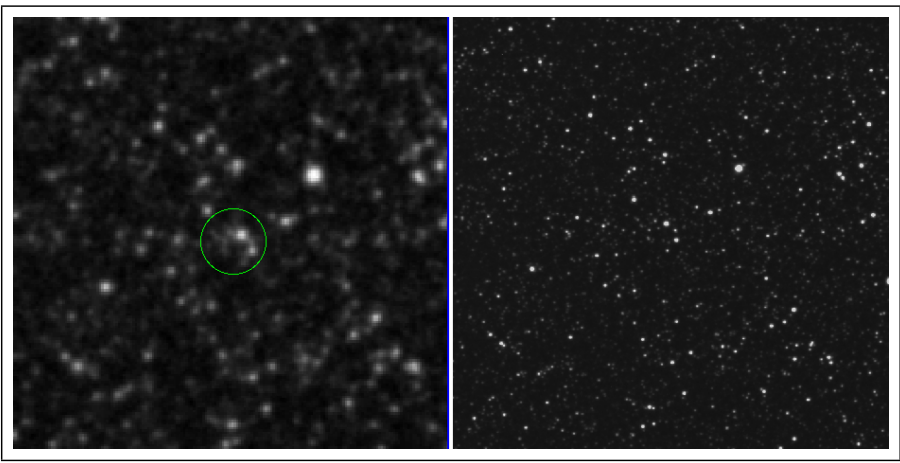

\section{OGLE-CL LMC 414}

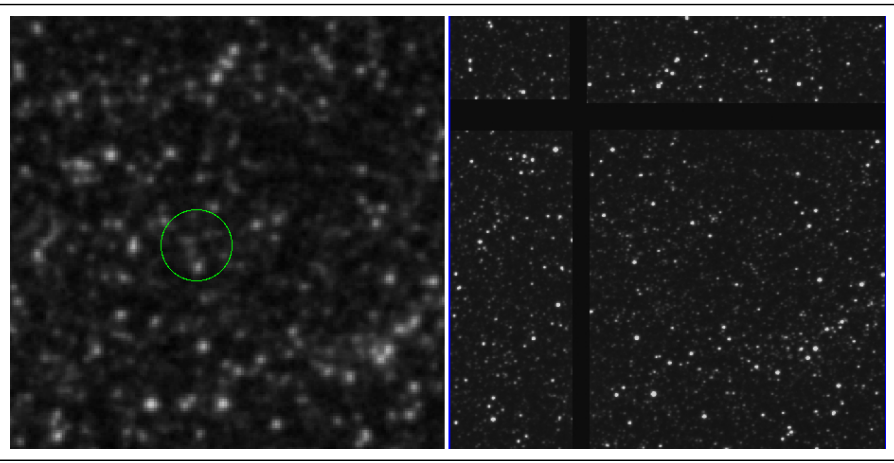

OGLE-CL LMC 425

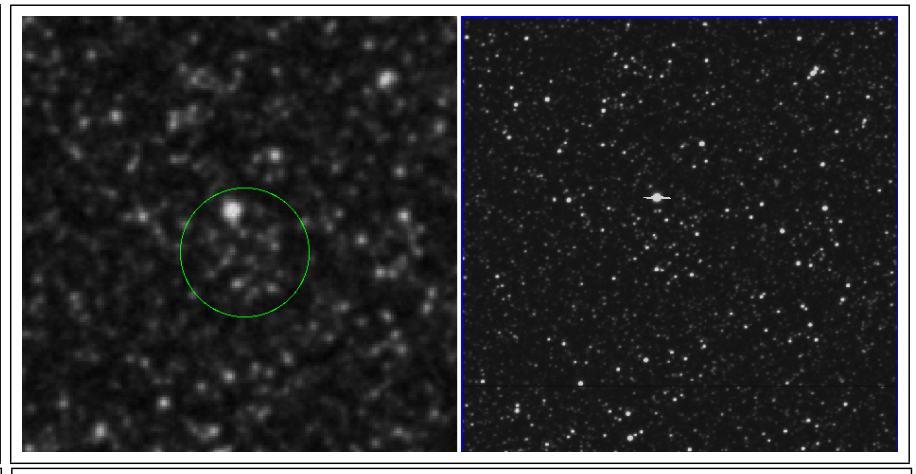

OGLE-CL LMC 406

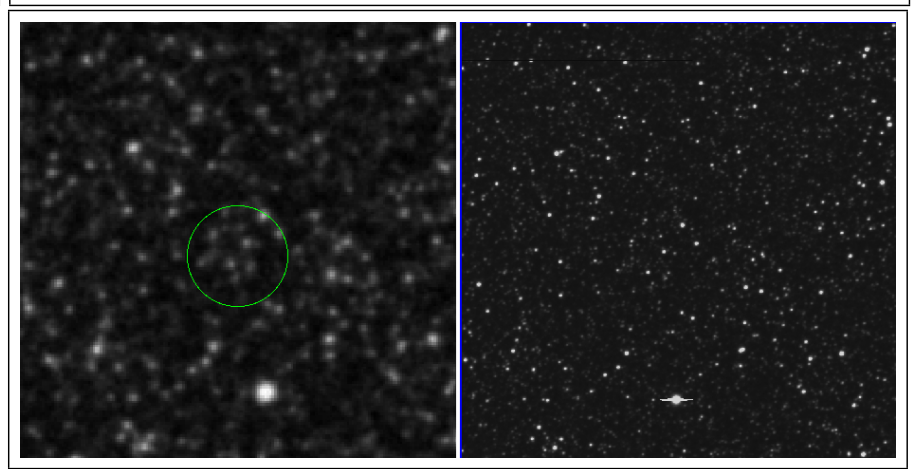

OGLE-CL LMC 412

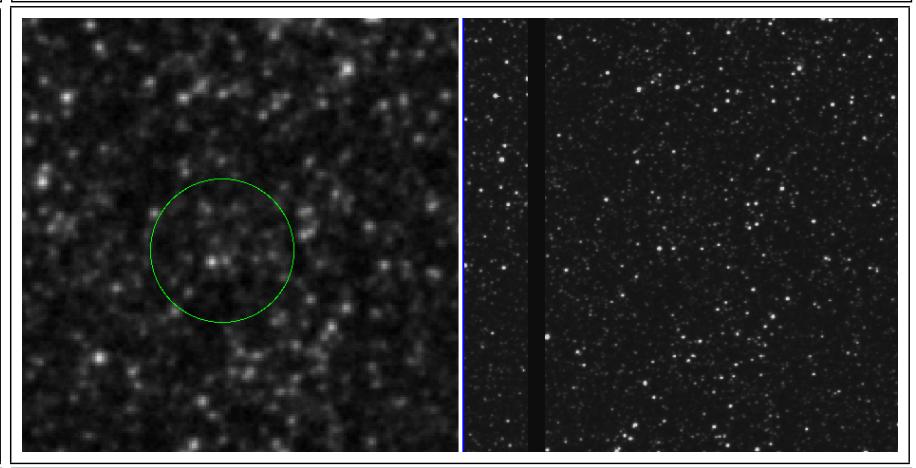

CL LMC 421

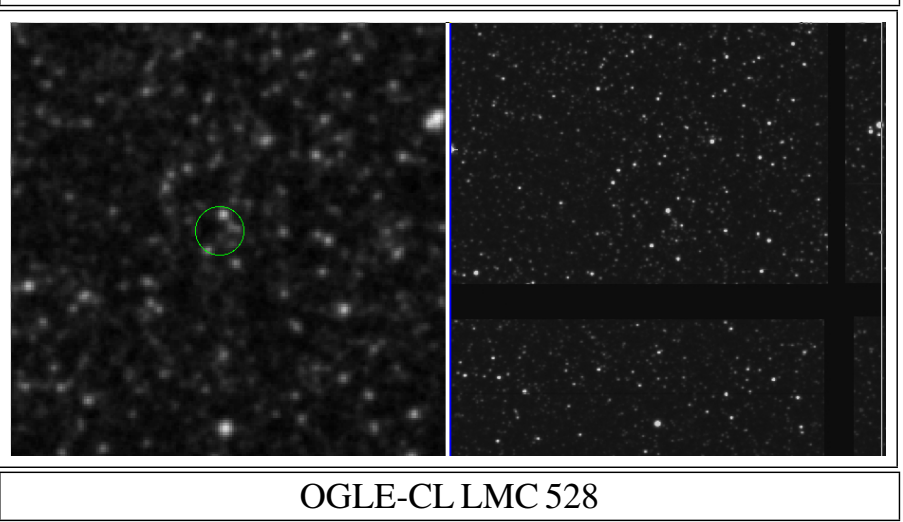

Fig. B.1. continued. 


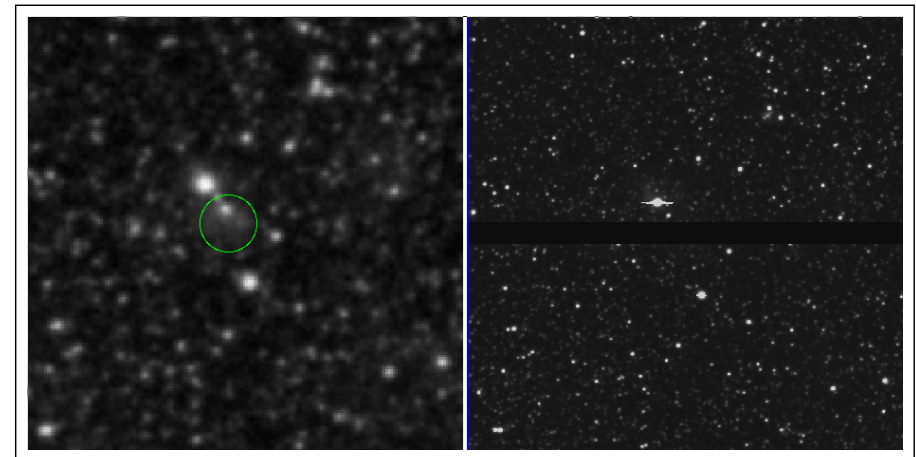

OGLE-CL LMC 430

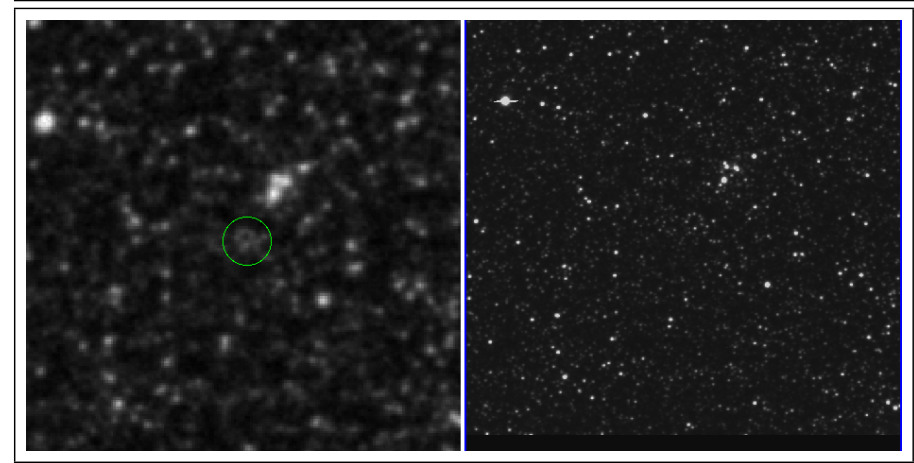

OGLE-CL LMC 434

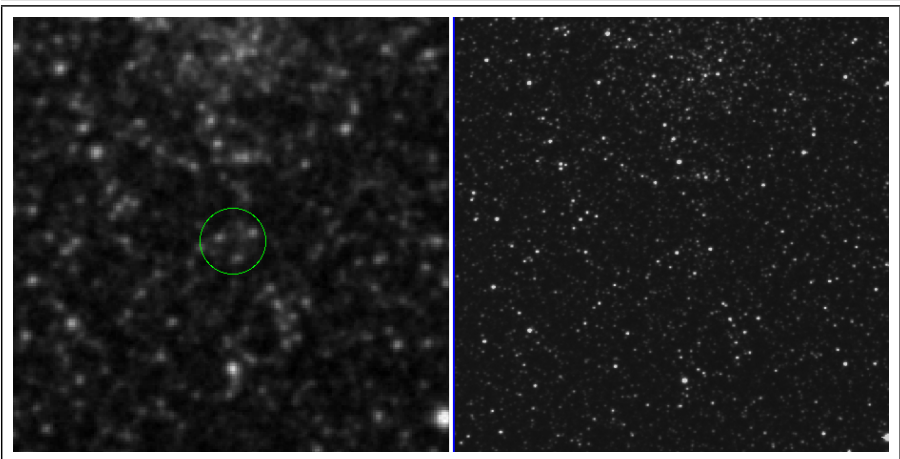

OGLE-CL LMC 437

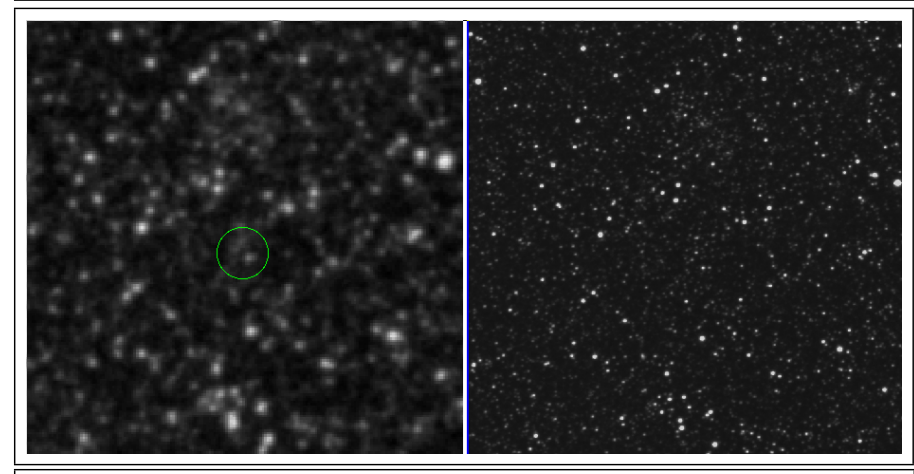

OGLE-CL LMC 441

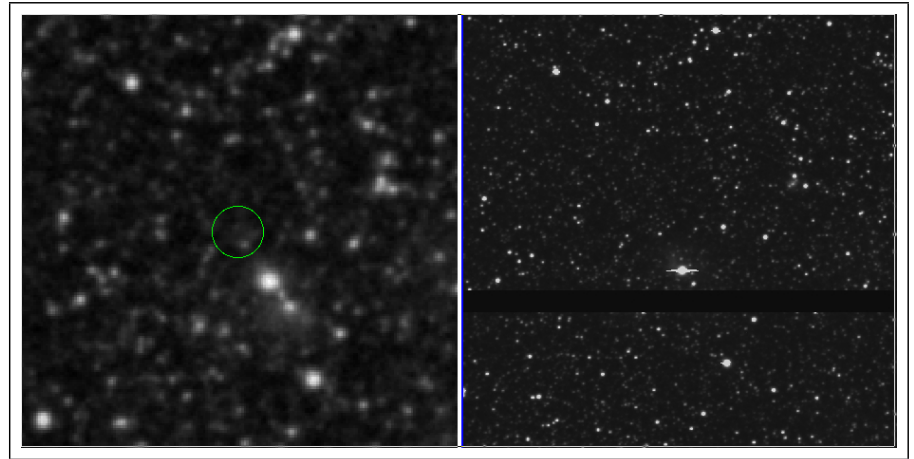

OGLE-CL LMC 433

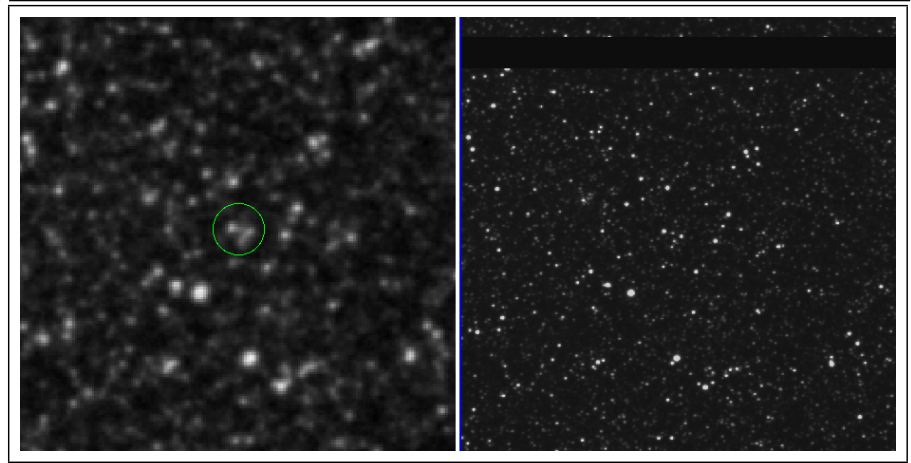

OGLE-CL LMC 435

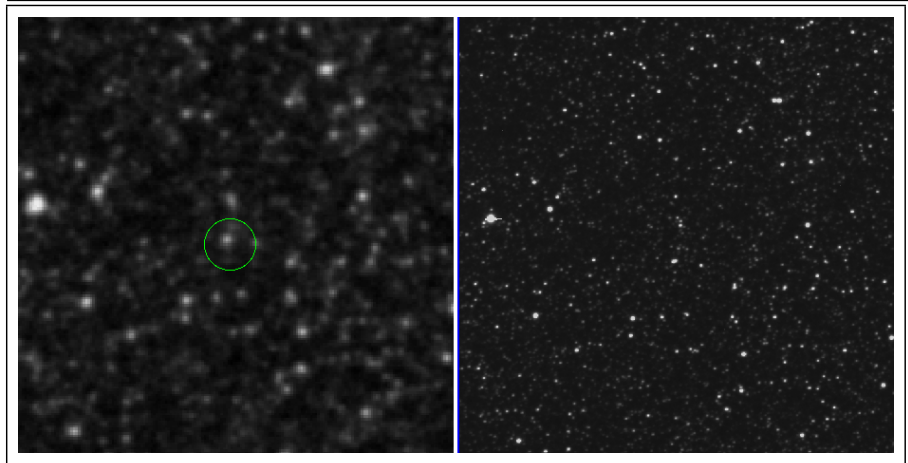

OGLE-CL LMC 439

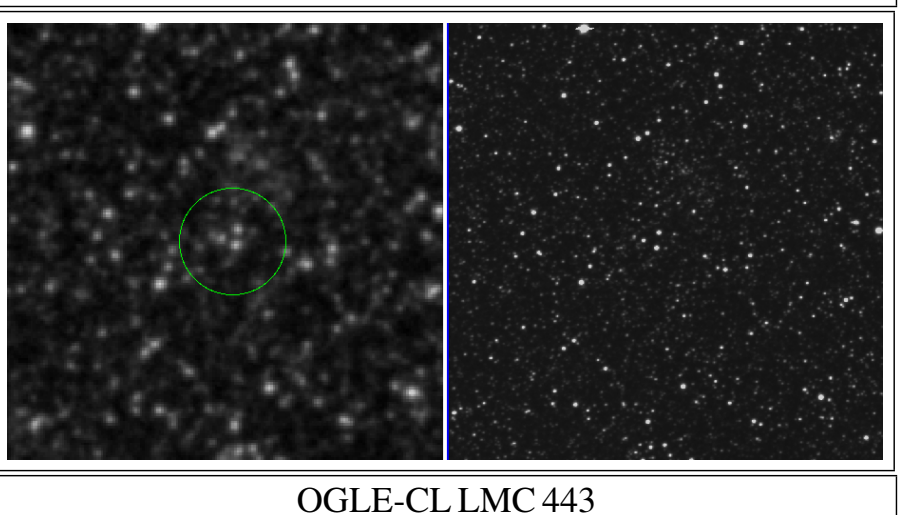

Fig. B.1. continued. 
A. E. Piatti: LMC bar star clusters

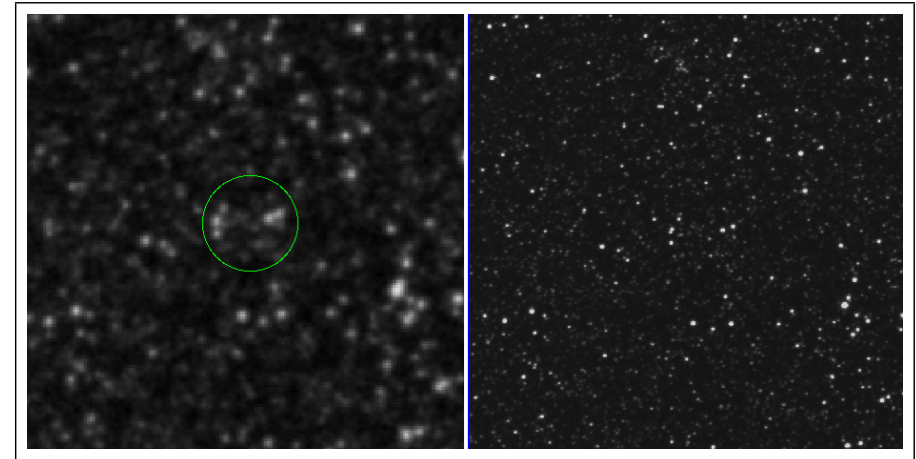

OGLE-CL LMC 448

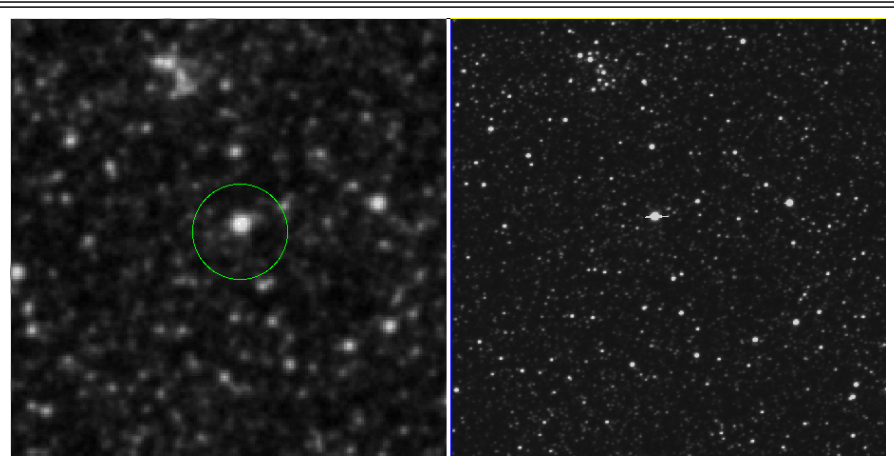

OGLE-CL LMC 465

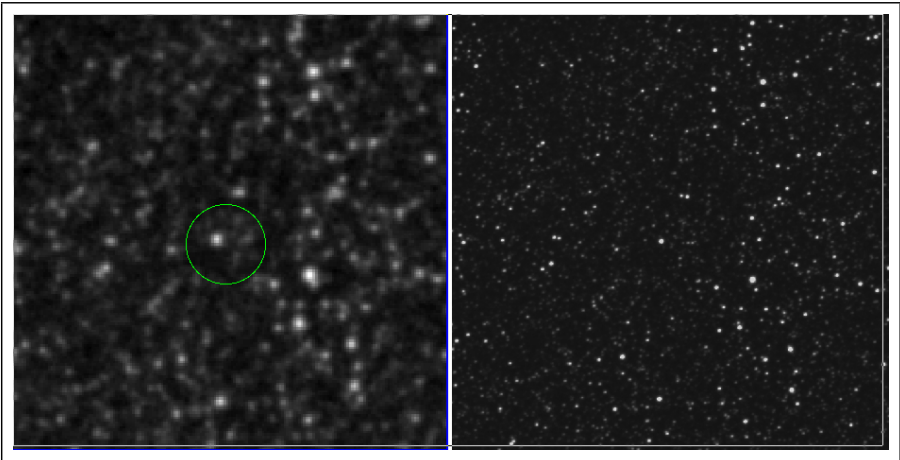

OGLE-CL LMC 474

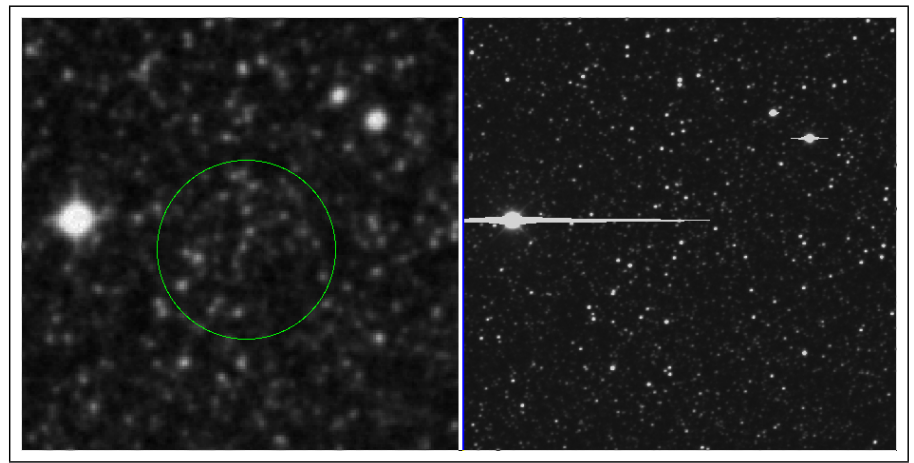

OGLE-CL LMC 455

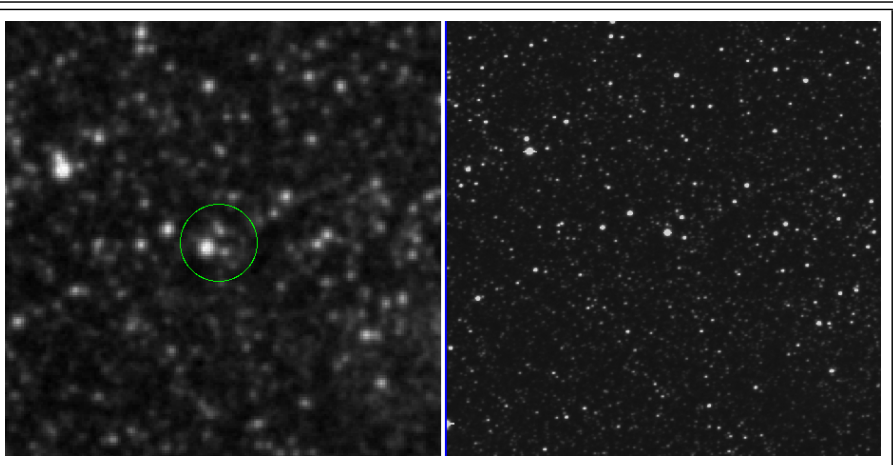

OGLE-CL LMC 466

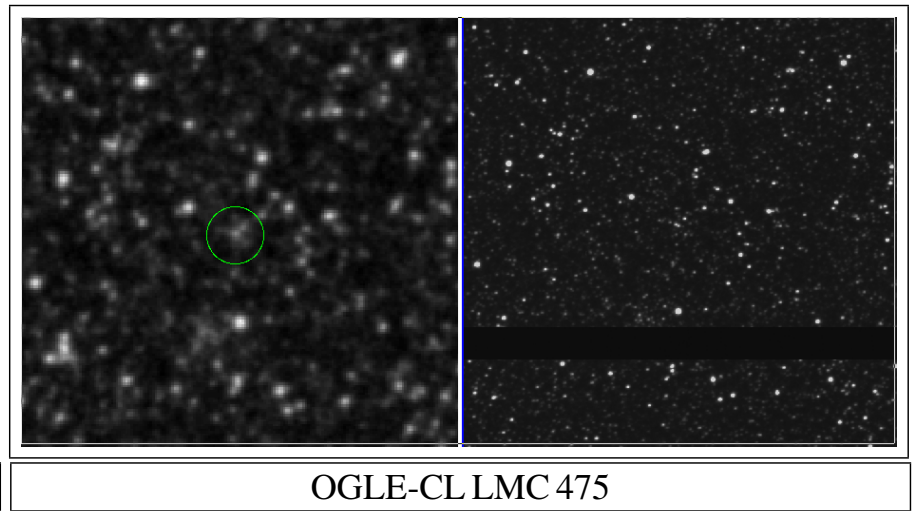

Fig. B.1. continued. 